\title{
Towards uniqueness of degenerate axially symmetric Killing horizon
}

\author{
Jacek Jezierski · Bartek Kamiński
}

Received: 13 October 2012 / Accepted: 3 February 2013 / Published online: 22 February 2013

(C) The Author(s) 2013. This article is published with open access at Springerlink.com

\begin{abstract}
For near horizon geometry we examine the linearized equations around extremal Kerr horizon (which is a unique axially symmetric near horizon geometry) and give some arguments towards stability of this horizon with respect to generic (nonsymmetric) linear perturbation of near horizon geometry. The result is also applicable for other situations like Kundt's class spacetimes or isolated horizons.
\end{abstract}

Keywords Near horizon geometry · Extremal Kerr horizon ·

Kundt's class spacetimes

\section{Introduction}

Let us consider the following basic equation on a two-dimensional compact manifold

$$
\omega_{A \| B}+\omega_{B \| A}+2 \omega_{A} \omega_{B}=R_{A B},
$$

where $\omega_{A} \mathrm{~d} x^{A}$ is a covector field, I denotes covariant derivative compatible with the metric $g_{A B}$, and $R_{A B}$ is its Ricci tensor. The Eq. (1) is a starting point of our considerations and it is a special case of (3.7) in [1], if we assume that $\tilde{S}_{A B}$ vanishes. See also [4] or [9].

\footnotetext{
J. Jezierski $(\varangle) \cdot$ B. Kamiński

Department of Mathematical Methods in Physics, Faculty of Physics, University of Warsaw, ul. Hoża 74, 00-682 Warsaw, Poland

e-mail: Jacek.Jezierski@fuw.edu.pl

B. Kamiński

e-mail: fizyk20@gmail.com
} 
Some geometric consequences of the basic equation ${ }^{1}$ (resulting from Einstein equations) were discussed in [6]. This is a non-linear PDE for unknown covector field and unknown Riemannian structure on the two-dimensional manifold. It appears in the context of Kundt's class metrics (cf. [7,8]), degenerate Killing horizons [4,9], or vacuum degenerate isolated horizons $[1,10,11]$. Several important results are already proved, like topological rigidity of the horizon and integrability conditions (cf. [6]). Moreover, when the one-form $\omega_{B} \mathrm{~d} x^{B}$ is closed (e.g. static degenerate horizon [4]) there are no solutions of (1). The transformation (4) of a covector $\omega_{A}$ leads to (partially) linear problem (invented in [6]) and simplifies the proof of the uniqueness of extremal Kerr for axially symmetric horizon. However, the problem of the existence of non-symmetric solutions to the basic equation remains open. The solutions of this equation enables one to construct near horizon metric (cf. [2-5,9]), Kundt's class spacetime or isolated horizon neighborhood.

In [6] the following results were proved:

Theorem 1 For any Riemannian metric $g_{A B}$ on a two-dimensional, compact, connected manifold $B$ with no boundary and genus $\mathbf{g} \geq 2$ there are no solutions of basic equation.

Theorem 2 For any Riemannian metric $g_{A B}$ on a two-dimensional torus Eq. (1) possesses only trivial solutions $\omega^{A} \equiv 0 \equiv K$ and the metric $g_{A B}$ is flat.

Theorem 3 There are no solutions of Eq. (1) with the following properties:

- $\omega^{A}=0$ only at finite set of points,

- $B$ is a sphere with non-negative Gaussian curvature.

The symmetric part of $\omega_{A|| B}$ is controlled by the equation but $f:=\frac{1}{2} \omega_{A|| B} \varepsilon^{A B}$ is an unknown function on a sphere. We have

$$
\omega_{A \| B}=f \varepsilon_{A B}+\frac{1}{2} K g_{A B}-\omega_{A} \omega_{B}
$$

The integrability condition:

$$
\frac{1}{4} R_{A}^{\| A}+2\left(R \omega^{A}\right)_{\| A}=6 f^{2}+\frac{3}{8} R\left(R-12 \omega_{A} \omega^{A}\right)
$$

implies that there exists non-empty open subset, where $12 \omega_{A} \omega^{A}>R>0$.

In this paper we analyze a linear perturbation of extremal Kerr solution. More precisely, in Sect. 2 we perform linearization of Eq. (1) around extremal Kerr solution (20). Axial symmetry of the background solution gives possibility to decompose linearized solution into Fourier series. Each Fourier mode $v_{k}$ fulfills ordinary differential Eq. (42). Using functional analysis methods we prove (in Appendix C) that there are no regular solutions for $|k|>8$. We hope to check numerically the nonexistence of low modes for $|k| \leq 8$.

\footnotetext{
1 This is an equation describing the so-called near horizon geometries see [2,3,5].
} 
Moreover, in this Section below we give some new results like equivalent formulations of the full nonlinear problem (cf. Theorem 4), equivalence between (2) and (18) or some properties contained in formulae at the end of Sect. 1.

Finally, some nontrivial calculations are shifted to the Appendix which also contains some useful formulae.

\subsection{Transformation to linear problem}

Let us denote

$$
\Phi_{A}:=\frac{\omega_{A}}{\omega^{B} \omega_{B}} .
$$

For any domain, where $\omega^{B} \omega_{B}>0$, equation (1) implies

$$
\Phi_{A \| C} \varepsilon^{A C}=\left(\frac{\omega_{A}}{\omega^{B} \omega_{B}}\right)_{\| C} \varepsilon^{A C}=0
$$

which simply means that the one-form $\Phi_{A} \mathrm{~d} x^{A}$ is closed, and locally there exists a coordinate $\Phi$ such that

$$
\mathrm{d} \Phi=\Phi_{A} \mathrm{~d} x^{A}
$$

Moreover, from (1) we get

$$
\Phi^{A}{ }_{\| A}=1
$$

hence the potential $\Phi$ is a solution of the Poisson's equation:

$$
\triangle \Phi=1
$$

Remark If we choose one isolated point, where $\omega$ vanishes, then for a given metric $g$ we have unique solution of the above Laplace-Beltrami equation (Green's function in the enlarged sense cf. [12]). For more isolated points we can take linear combination of such solutions. More precisely, let $G_{x_{0}}$ be a unique solution (for a given metric $g$ ) of the Eq. (7) on $S^{2}-\left\{x_{0}\right\}$. If $c_{0}+c_{1}+\ldots+c_{n}=1$ (where $c_{i} \in \mathbb{R}$ ) then $\Phi=c_{0} G_{x_{0}}+c_{1} G_{x_{1}}+\ldots+c_{n} G_{x_{n}}$ is a solution of (7) on $S^{2}-\left\{x_{0}, x_{1}, \ldots, x_{n}\right\}$, and $\omega$ vanishes at the points $x_{0}, x_{1}, \ldots, x_{n}$.

\subsection{Two zeros of $\omega$}

Suppose $\omega_{A}$ vanishes at two distinct points in a generic way (i.e. $\omega_{A \| B}$ is nondegenerate at those points). Then the Eqs. (6) and (5) extend (in the sense of distributions) as follows: 


$$
\begin{gathered}
\Phi_{\| A}^{A}=1-c_{1} \boldsymbol{\delta}_{\theta=\pi}-c_{2} \boldsymbol{\delta}_{\theta=0}, \\
\Phi_{A \| C} \varepsilon^{A C}=d_{1} \boldsymbol{\delta}_{\theta=\pi}-d_{2} \boldsymbol{\delta}_{\theta=0} .
\end{gathered}
$$

Integration of the above equations on $S^{2}$ implies $d_{1}=d_{2}=d$ and $c_{1}+c_{2}=$ $\int \lambda=\left(\right.$ total volume of $S^{2}$ ). Hence, for $\Phi_{A}=\partial_{A} \Phi+\varepsilon_{A}{ }^{B} \partial_{B} \tilde{\Phi}$ the potentials $\Phi, \tilde{\Phi}$ fulfill Laplace equations:

$$
\begin{aligned}
& \triangle \Phi=1-c_{1} \boldsymbol{\delta}_{\theta=\pi}-c_{2} \boldsymbol{\delta}_{\theta=0}, \\
& \triangle \tilde{\Phi}=d_{1} \boldsymbol{\delta}_{\theta=\pi}-d_{2} \boldsymbol{\delta}_{\theta=0},
\end{aligned}
$$

and their solutions may be expressed in terms of generalized Green's functions on $S^{2}$ which are well defined as the distributions (they are integrable functions, smooth outside poles with log divergence at poles).

Moreover, the trace of (1)

$$
\omega_{\| A}^{A}=K-\omega^{A} \omega_{A}
$$

may be expressed in terms of $\Phi^{A}$ as follows:

$$
\partial_{A}\left(\frac{\lambda \Phi^{A}}{\Phi^{B} \Phi_{B}}\right)+\frac{\lambda}{\Phi^{B} \Phi_{B}}-\lambda K=0 \equiv \frac{2}{\|\Phi\|} \partial_{A}\left(\frac{\lambda \Phi^{A}}{\|\Phi\|}\right)=\lambda K .
$$

1.3 Equivalent form of the basic equation in terms of the covector $\Phi_{A}$ and its conformal rescaling

Equations (5) and (6) together with (13) written as follows:

$$
\begin{gathered}
\lambda \varepsilon^{A C} \partial_{C} \Phi_{A}=0, \\
\partial_{A}\left(\lambda g^{A B} \Phi_{B}\right)=\lambda, \\
\partial_{A}\left(\frac{\lambda g^{A B} \Phi_{B}}{g^{C D} \Phi_{C} \Phi_{D}}\right)+\frac{\lambda}{g^{C D} \Phi_{C} \Phi_{D}}-\lambda K=0,
\end{gathered}
$$

for the conformally equivalent metric $h_{A B}=\exp (-2 u) g_{A B}$ (cf. Eq. (22)) are almost the same

$$
\begin{aligned}
& (\lambda K)(h)-(\lambda K)(g)=\lambda_{h} \triangle_{h} u=\lambda_{g} \triangle_{g} u, \quad-(\lambda K)(h)=\frac{1}{2} a^{2}, x x, \\
& \partial_{A}\left(\lambda_{h} h^{A B} \Phi_{B}\right)=\lambda_{h} \exp (2 u), \quad \lambda \varepsilon^{A C} \partial_{C} \Phi_{A}=0, \\
& \partial_{A}\left(\frac{\lambda_{h} h^{A B} \Phi_{B} \exp (2 u)}{h^{C D} \Phi_{C} \Phi_{D}}\right)+\frac{\lambda \exp (4 u)}{h^{C D} \Phi_{C} \Phi_{D}}-\lambda_{h} K_{h}+\lambda_{h} \triangle_{h} u=0 .
\end{aligned}
$$

Moreover, we have the following 
Theorem 4 Equations (14-16) are locally equivalent to the Eq. (2) in the domain, where $\omega_{A}=\frac{\Phi_{A}}{\Phi^{B} \Phi_{B}}$ is not vanishing.

Proof Let us represent tensor $\omega_{A \| B}$ as a sum of three parts: skewsymmetric $(f)$, traceless symmetric $\left(\tau_{A B}\right)$ and trace $(\tau)$ :

$$
\omega_{A \| B}=f \varepsilon_{A B}+\tau_{A B}+\tau g_{A B} .
$$

We have to show that $\tau_{A B}$ and $\tau$ are determined by Eq. (14-16). It is easy to check that (16) implies $2 \tau=K-\|\omega\|^{2}=\omega^{A} \| A$. Moreover, (14) gives

$$
\varepsilon^{A B} \omega_{A} \omega^{C} \tau_{C B}=0
$$

and similarly (15) implies

$$
2 \omega^{A} \omega^{B} \tau_{A B}=-\|\omega\|^{4}
$$

Let us observe that any two-dimensional traceless symmetric tensor has only two independent components, hence the last two conditions determine $\tau_{A B}$ uniquely in the following form:

$$
\tau_{A B}=-\omega_{A} \omega_{B}+\frac{1}{2} g_{A B}\|\omega\|^{2} .
$$

Finally, the above formula together with $\tau=\frac{1}{2}\left(K-\|\omega\|^{2}\right)$ give the Eq. (2).

One can also check the following formula:

$$
\begin{aligned}
\Phi_{A \| B}= & 0 \cdot \varepsilon_{A B}+\frac{1}{2} g_{A B}-f\left(* \Phi_{A} \Phi_{B}+* \Phi_{B} \Phi_{A}\right) \\
& +\left(1-K\|\Phi\|^{2}\right)\left(\frac{\Phi_{A} \Phi_{B}}{\|\Phi\|^{2}}-\frac{1}{2} g_{A B}\right)
\end{aligned}
$$

which is equivalent to (2) but in terms of $\Phi$.

Let us observe that $\Phi^{B}{ }_{\| B A}=0$ hence the symmetry of the tensor $\Phi_{A \| B}$ implies

$$
\Phi_{A}{ }_{B B}=\Phi^{B}{ }_{\| A B}=\Phi^{B}{ }_{\| A B}-\Phi^{B}{ }_{\| B A}=R_{A B} \Phi^{B}
$$

and we obtain the following nice formulae:

$$
\Phi^{A \| B}{ }_{B}=K \Phi^{A}, \quad * \Phi^{A \| B}{ }_{B}=K * \Phi^{A} .
$$

Moreover,

$$
\Phi^{A \| B} \omega_{A \| B}=K-\|\omega\|^{2}
$$


and

$$
\begin{aligned}
& \left(\Phi^{A \| B} \omega_{A}\right)_{\| B}=\Phi_{A}\left\|B{ }_{B} \omega^{A}+\Phi^{A \| B} \omega_{A \| B}=2 K-\right\| \omega \|^{2}, \\
& \lim _{\epsilon \rightarrow 0^{+}} \int_{\partial S_{\epsilon}} \Phi^{A \| B} \omega_{A} \mathrm{~d} S_{B}=\int_{S^{2}} K=4 \pi, \quad \text { where } S_{\epsilon}:=S^{2} \backslash\left(\bigcup_{x_{i} \in \omega^{-1}(\{0\})} K\left(x_{i}, \epsilon\right)\right) .
\end{aligned}
$$

\section{Linearization of basic equation around extremal Kerr}

After introducing a new coordinate $x:=\cos \theta$ the (two-dimensional) extremal Kerr (see [6])

$$
\begin{aligned}
g_{\text {Kerr }} & =2 m^{2}\left[\frac{1+\cos ^{2} \theta}{2} \mathrm{~d} \theta^{2}+\frac{2 \sin ^{2} \theta}{1+\cos ^{2} \theta} \mathrm{d} \phi^{2}\right], \\
\omega^{\theta} & =-\frac{\sin \theta \cos \theta}{m^{2}\left(1+\cos ^{2} \theta\right)^{2}}, \quad \omega^{\varphi}=\frac{1}{2 m^{2}\left(1+\cos ^{2} \theta\right)},
\end{aligned}
$$

takes the following form:

$$
g_{\text {Kerr }}=h_{A B} \mathrm{~d} x^{A} \mathrm{~d} x^{B}=2 m^{2}\left(a^{-2} \mathrm{~d} x^{2}+a^{2} \mathrm{~d} \varphi^{2}\right),
$$

where $a^{2}:=2 \frac{1-x^{2}}{1+x^{2}}$ and $\lambda:=\sqrt{\operatorname{det} h_{A B}}=2 m^{2}$. The components of various objects for Kerr are the following:

$$
\begin{gathered}
\omega_{x}=\frac{x}{1+x^{2}}, \quad \omega_{\varphi}=\frac{a^{2}}{1+x^{2}}, \quad\|\omega\|^{2}=\frac{1}{2 m^{2}} \frac{a^{2}}{1+x^{2}} . \\
\frac{1}{2 m^{2}} \Phi=\frac{x}{a^{2}} \mathrm{~d} x+\mathrm{d} \varphi, \quad \frac{1}{2 m^{2}} * \Phi=\frac{1}{a^{2}} \mathrm{~d} x-x \mathrm{~d} \varphi \quad\left(* \Phi_{A}:=\varepsilon_{A}{ }^{B} \Phi_{B}\right), \\
K=\frac{2}{m^{2}} \frac{1-3 x^{2}}{\left(1+x^{2}\right)^{3}}, \quad f=\frac{1}{m^{2}} \frac{x\left(1+a^{2}\right)}{\left(1+x^{2}\right)^{2}}=\frac{1}{m^{2}} \frac{x\left(3-x^{2}\right)}{\left(1+x^{2}\right)^{3}}, \\
\frac{K}{2}+i f=\frac{1}{m^{2}(1-i x)^{3}}, \\
\|\Phi\|=\|* \Phi\|, \quad * \Phi \wedge \Phi=\|\Phi\|^{2} \lambda \mathrm{d} x \wedge \mathrm{d} \varphi .
\end{gathered}
$$

The nearby metric $g$ we describe by conformal factor:

$$
g_{A B}=\exp (2 u) h_{A B}
$$

and we get

$$
\begin{aligned}
\Gamma_{A B}^{C}(g) & =\Gamma_{A B}^{C}(h)+S_{A B}^{C}, \\
S_{A B}^{C} & =\delta_{A}^{C} \partial_{B} u+\delta_{B}^{C} \partial_{A} u-h_{A B} h^{C D} \partial_{D} u .
\end{aligned}
$$


Let us denote by $u^{B}:=h^{B A} \partial_{A} u$ the gradient of $u$ with respect to the metric $h$. We have

$$
\begin{aligned}
\nabla_{B}(g) \omega_{A} & =\nabla_{B}(h) \omega_{A}-S_{A B}^{C}(u) \omega_{C} \\
& =\nabla_{B}(h) \omega_{A}+h_{A B} \omega_{C} u^{C}-\omega_{A} u_{B}-\omega_{B} u_{A} .
\end{aligned}
$$

Moreover, the Gaussian curvatures $K_{h}$ and $K_{g}$ for the conformally related metrics $h$ and $g$ respectively are related as follows

$$
\triangle_{h} u=K_{h}-\exp (2 u) K_{g}
$$

This gives the following transformation for the right-hand side of (1):

$$
R_{A B}(g)=K_{g} g_{A B}=\left(K_{h}-\triangle_{h} u\right) h_{A B} .
$$

Using (27) and (29) we rewrite basic Eq. (1) as follows:

$$
\begin{aligned}
& \nabla_{B}(h) \omega_{A}+\nabla_{A}(h) \omega_{B}+2\left(h_{A B} \omega_{C} u^{C}-\omega_{A} u_{B}-\omega_{B} u_{A}+\omega_{A} \omega_{B}\right) \\
& =\left(K_{h}-\triangle_{h} u\right) h_{A B} .
\end{aligned}
$$

Let us denote the linear part of the covector $\omega$ by

$$
\mathrm{w}_{A}:=\omega_{A}-\omega_{A}^{\mathrm{Kerr}}
$$

Now we are ready to linearize basic equation.

$$
\begin{aligned}
& 2\left(\omega_{A}^{\mathrm{Kerr}} \mathbf{w}_{B}+\omega_{B}^{\mathrm{Kerr}} \mathbf{w}_{A}+h_{A B} \omega_{C}^{\mathrm{Kerr}} u^{C}-\omega_{A}^{\mathrm{Kerr}} u_{B}-\omega_{B}^{\mathrm{Kerr}} u_{A}\right)+\nabla_{B}(h) \mathbf{w}_{A} \\
& \quad+\nabla_{A}(h) \mathbf{w}_{B}+h_{A B} \triangle_{h} u=2 \mathbf{w}_{A} u_{B}+2 \mathbf{w}_{B} u_{A}-2 h_{A B} \mathbf{w}_{C} u^{C}-2 \mathbf{w}_{A} \mathbf{w}_{B} \approx 0 .
\end{aligned}
$$

Finally, for covector $\mathrm{w}_{A}$ and conformal factor $u$ in (26) the linearization of (1) takes the following form:

$$
\begin{aligned}
& \nabla_{A}\left(\mathrm{w}^{A}+u^{A}\right)+2 \omega^{A} \mathrm{w}_{A}=0, \\
& T S\left(\nabla_{A} \mathbf{w}_{B}+2 \omega_{A}\left(\mathrm{w}_{B}-u_{B}\right)\right)=0,
\end{aligned}
$$

where now $\omega$ and $\nabla$ are background objects (corresponding to the Kerr solution (22)), and

$$
T S\left(t_{A B}\right):=t_{A B}+t_{B A}-h_{A B} h^{C D} t_{C D}
$$

denotes the traceless symmetric part of the tensor $t_{A B}$. 
We show in Appendix B that after elimination of $u_{A}$ we get:

$$
\begin{aligned}
& \triangle_{h}\left(\mathrm{w}_{A} * \Phi^{A}\right)+\varepsilon^{A B} \mathrm{w}_{A \| B}=0, \\
& \triangle_{h}\left(\mathrm{w}_{A} \Phi^{A}\right)+4 \mathrm{w}_{A} \Phi^{A}\|\omega\|^{2}+3 \mathrm{w}_{\| A}^{A}=0
\end{aligned}
$$

where

$$
u_{A}=\frac{1}{2}\left[\mathbf{w}_{A}+\nabla_{B}\left(\Phi^{B} \mathbf{w}_{A}-\Phi_{A} \mathbf{w}^{B}\right)+\nabla_{A}\left(\Phi^{B} \mathbf{w}_{B}\right)\right] .
$$

Remark The Eqs. (34-35) are conformally covariant with respect to the rescaling of the two-metric $h$. More precisely, the form of these equations is the same for two conformally related metrics provided that $\Phi, * \Phi$ are vector fields and $\mathrm{w}$ and $\omega$ are covector fields. One can easily verify this observation multiplying the above equations by scalar density $\lambda$.

The non-existence of the solution $\mathrm{w}_{A}$ to the Eqs. (34-35) is equivalent to the stability of the solution (20-21).

Axial symmetry of the background solution enables one to separate variable $\varphi$ with the help of Fourier transform and (34-35) becomes second order ODE for

$$
\mathrm{w}:[-1,1] \mapsto \mathbb{R}^{2} .
$$

One can also introduce another pair of variables:

$$
\begin{aligned}
& \alpha:=\varepsilon^{A B} \mathrm{w}_{A} \Phi_{B}=\frac{1}{2}\left[2 \mathrm{w}_{x}-\frac{x\left(1+x^{2}\right)}{1-x^{2}} \mathbf{w}_{\varphi}\right]=\mathrm{w}_{x}-\frac{x\left(1+x^{2}\right)}{2\left(1-x^{2}\right)} \mathbf{w}_{\varphi}=\mathrm{w}_{x}-\frac{x}{a^{2}} \mathbf{w}_{\varphi}, \\
& \beta:=\Phi_{A} \mathbf{w}^{A}=m^{2}\left[\frac{x\left(1+x^{2}\right)}{1-x^{2}} \mathbf{w}^{x}+2 \mathbf{w}^{\varphi}\right]=x \mathbf{w}_{x}+\frac{1+x^{2}}{2\left(1-x^{2}\right)} \mathbf{w}_{\varphi}=x \mathbf{w}_{x}+\frac{1}{a^{2}} \mathbf{w}_{\varphi},
\end{aligned}
$$

where $a^{2}:=2 \frac{1-x^{2}}{1+x^{2}}$. The formula (36) takes a simple form:

$$
u_{A}=\frac{1}{2}\left[\mathrm{w}_{A}+\varepsilon_{A}^{B} \nabla_{B} \alpha+\nabla_{A} \beta\right] .
$$

Moreover, the inverse transformation

$$
\mathrm{w}_{x}=\frac{\alpha+x \beta}{1+x^{2}}, \quad \mathbf{w}_{\varphi}=a^{2} \frac{\beta-x \alpha}{1+x^{2}},
$$

implies the following form of the Eqs. (34-35) in terms of variables $\alpha, \beta$ :

$$
\begin{aligned}
& \triangle_{h}(\alpha)+\partial_{\varphi}\left(\frac{\alpha+x \beta}{1+x^{2}}\right)-\partial_{x}\left(a^{2} \frac{\beta-x \alpha}{1+x^{2}}\right)=0, \\
& \triangle_{h}(\beta)+\frac{4 a^{2}}{1+x^{2}} \beta+3 \partial_{\varphi}\left(\frac{\beta-x \alpha}{1+x^{2}}\right)+3 \partial_{x}\left(a^{2} \frac{\alpha+x \beta}{1+x^{2}}\right)=0,
\end{aligned}
$$


where

$$
\triangle_{h}:=\partial_{x} a^{2} \partial_{x}+\partial_{\varphi} a^{-2} \partial_{\varphi}
$$

Let us denote $v:=\left[\begin{array}{l}\alpha \\ \beta\end{array}\right], B:=\frac{1}{1+x^{2}}\left[\begin{array}{ll}x & -1 \\ 3 & 3 x\end{array}\right], C:=\frac{1}{1+x^{2}}\left[\begin{array}{cc}1 & x \\ -3 x & 3\end{array}\right]$, then the Eqs. (39-40) take the following (matrix) form:

$$
\triangle_{h} v+\frac{4 a^{2}}{1+x^{2}}\left[\begin{array}{ll}
0 & 0 \\
0 & 1
\end{array}\right] v+\partial_{x}\left(a^{2} B v\right)+\partial_{\varphi}(C v)=0
$$

Other useful identities:

$$
\begin{aligned}
\mathbf{w}_{A} & =\beta \omega_{A}+\alpha \varepsilon_{A}{ }^{B} \omega_{B}=\partial_{A}(2 u-\beta)-\varepsilon_{A}^{B} \partial_{B} \alpha, \\
2 \partial_{A} u & =\partial_{A} \beta+\beta \omega_{A}+\varepsilon_{A}^{B}\left(\partial_{B} \alpha+\alpha \omega_{B}\right) .
\end{aligned}
$$

\subsection{Boundary data}

A small perturbation of Kerr data (20-21) does not destroy the number of two zeros for covector $\omega_{A}$. This is a simple consequence of the "inverse function theorem". More precisely, the non-vanishing curvature in the neighborhood of "spherical pole" (zero of $\omega_{A}$ ) assures invertibility of the first derivative $\nabla_{A} \omega_{B}$ in a small open neighborhood ${ }^{2}$ and implies existence of a local diffeomorphism $\omega_{A}\left(x^{B}\right)$. Hence, for perturbed $\omega_{A}\left(x^{B}\right)$ there exists (in a small open neighborhood of spherical pole) precisely one point, where $\omega_{A}$ vanishes. The freedom of global conformal transformations enables one to introduce "new conformal coordinates" in such a way that the spherical poles are always at the points where $\omega_{A}$ vanishes. Hence, we can always assume that the perturbed $\omega_{A}$ vanishes at spherical poles which implies zero (homogeneous) boundary data for linear perturbation $\mathrm{w}_{A}$ or equivalently for $v=(\alpha, \beta)^{3}$.

One can also show that respectively chosen conformal vector field $X$ enables one to change $\mathrm{w}_{A} \rightarrow \mathrm{w}_{A}+\mathcal{L}_{X} \omega_{A}$ in such a way that it will vanish at a given point (see Appendix D).

Hypothesis The Eq. (41) has no regular solutions for homogeneous boundary data $\left.\mathrm{w}_{A}\right|_{x=1}=0=\left.\mathrm{w}_{A}\right|_{x=-1}$.

Proof attempt Let us consider Fourier series for $v$ :

$$
v(x, \phi)=\sum_{k=-\infty}^{\infty} v_{k}(x) e^{i k \phi}
$$

\footnotetext{
2 Formula (2) implies that $\operatorname{det} \nabla_{A} \omega^{B}=f^{2}+\frac{K}{2}\left(\frac{K}{2}-\|\omega\|^{2}\right)$, for Kerr $\operatorname{det} \nabla_{A} \omega^{B}=\frac{2 x^{2}\left(3-x^{2}\right)}{\left(1+x^{2}\right)^{5}}$ and it vanishes only on the equator $x=0$.

${ }^{3}$ It is not obvious that $\mathrm{w}_{A}=0$ corresponds to $v=0$ and it is not true for $k=0$.
} 
It leads to ODE for $v_{k}(x)$ :

$$
\partial_{x} a^{2} \partial_{x} v_{k}-\frac{k^{2}}{a^{2}} v_{k}+\frac{4 a^{2}}{1+x^{2}}\left[\begin{array}{ll}
0 & 0 \\
0 & 1
\end{array}\right] v_{k}+\partial_{x}\left(a^{2} B v_{k}\right)+i k\left(C v_{k}\right)=0 .
$$

We check that $v_{k}$ vanishes at poles for $|k| \geq 1$, because $\mathrm{w}_{A}$ vanishes there. For $k=0$ we have axial symmetry, hence we already have uniqueness in full nonlinear case, however it would be nice to check this fact independently.

For $|k|>8$ we show in Appendix $\mathrm{C}$ that there are no regular solutions. There are some initial numerical results which confirm nonexistence hypothesis for $|k| \leq 8$. We are going to check numerically the existence or nonexistence of low modes. The results will be published in a separate paper.

The above Hypothesis implies stability of the extremal Kerr horizon. It is true for $|k|>8$ and adding this assumption we get Theorem 5. More precisely, Eq. (42) has no regular solutions for $|k|>8$. The analytical proof is given in Appendix C.

Acknowledgments This research was supported by Polish Ministry of Science and Higher Education grant Nr N N201 372736.

Open Access This article is distributed under the terms of the Creative Commons Attribution License which permits any use, distribution, and reproduction in any medium, provided the original author(s) and the source are credited.

\section{Appendix A: Kerr in conformal coordinates}

The background metric (22) can be conformally related to unit sphere metric as follows:

$$
g_{\text {Kerr }}=h_{A B} \mathrm{~d} x^{A} \mathrm{~d} x^{B}=2 m^{2}\left(a^{-2} \mathrm{~d} x^{2}+a^{2} \mathrm{~d} \varphi^{2}\right)=2 m^{2} F^{2} \tilde{h}_{A B} \mathrm{~d} \tilde{x}^{A} \mathrm{~d} \tilde{x}^{B},
$$

where

$$
\tilde{h}_{A B} \mathrm{~d} \tilde{x}^{A} \mathrm{~d} \tilde{x}^{B}:=\left[\frac{\mathrm{d} \tilde{x}^{2}}{1-\tilde{x}^{2}}+\left(1-\tilde{x}^{2}\right) \mathrm{d} \tilde{\varphi}^{2}\right], \quad \tilde{\varphi}=\varphi, \quad \tilde{x}=\frac{x-\tanh \frac{x}{2}}{1-x \tanh \frac{x}{2}}
$$

is the usual unit sphere metric and

$$
F^{2}=\frac{a^{2}}{1-\tilde{x}^{2}}=\frac{2}{1+x^{2}}\left(\cosh \frac{x}{2}-x \sinh \frac{x}{2}\right)^{2}, \quad \mathrm{~d} x=F^{2} \mathrm{~d} \tilde{x} .
$$




\section{Appendix B: Reduced linearized equations}

\section{B.1 Elimination of $u_{A}$}

We start from traceless part (33):

$$
\begin{aligned}
& \nabla_{A} \mathbf{w}_{B}+\nabla_{B} \mathbf{w}_{A}+2 \omega_{A} \mathbf{w}_{B}+2 \omega_{B} \mathbf{w}_{A}-\nabla^{C} \mathbf{w}_{C} h_{A B}-2 \omega^{C} \mathbf{w}_{C} h_{A B}+ \\
& -2 \omega_{A} u_{B}-2 \omega_{B} u_{A}+2 \omega^{C} u_{C} h_{A B}=0 .
\end{aligned}
$$

The two independent components $(A B)=(x x)$ and $(A B)=(x \phi)$ can be written as follows. Component $(x x)$ :

$$
\begin{aligned}
& 2 \nabla_{x} \mathbf{w}_{x}+4 \omega_{x} \mathbf{w}_{x}-\left(\nabla^{x} \mathbf{w}_{x}+\nabla^{\phi} \mathbf{w}_{\phi}\right) h_{x x}-2\left(\omega^{x} \mathbf{w}_{x}+\omega^{\phi} \mathbf{w}_{\phi}\right) h_{x x}+ \\
& -4 \omega_{x} u_{x}+2\left(\omega^{x} u_{x}+\omega^{\phi} u_{\phi}\right) h_{x x}=0
\end{aligned}
$$

or in an equivalent form (dividing by $h_{x x}$ ):

$$
\begin{aligned}
& 2 \nabla^{x} \mathbf{w}_{x}+4 \omega^{x} \mathbf{w}_{x}-\nabla^{x} \mathbf{w}_{x}-\nabla^{\phi} \mathbf{w}_{\phi}-2 \omega^{x} \mathbf{w}_{x}-2 \omega^{\phi} \mathbf{w}_{\phi}-4 \omega^{x} u_{x}+2 \omega^{x} u_{x}+ \\
& 2 \omega^{\phi} u_{\phi}=0, \\
& 2 \omega^{x} u_{x}-2 \omega^{\phi} u_{\phi}=\nabla^{x} \mathbf{w}_{x}-\nabla^{\phi} \mathbf{w}_{\phi}+2 \omega^{x} \mathbf{w}_{x}-2 \omega^{\phi} \mathbf{w}_{\phi} .
\end{aligned}
$$

Component $(x \phi)$ :

$$
\begin{aligned}
& \nabla_{x} \mathbf{w}_{\phi}+\nabla_{\phi} \mathbf{w}_{x}+2 \omega_{x} \mathbf{w}_{\phi}+2 \omega_{\phi} \mathbf{w}_{x}-2 \omega_{x} u_{\phi}-2 \omega_{\phi} u_{x}=0, \\
& 2 \omega_{\phi} u_{x}+2 \omega_{x} u_{\phi}=\nabla_{x} \mathbf{w}_{\phi}+\nabla_{\phi} \mathbf{w}_{x}+2 \omega_{x} \mathbf{w}_{\phi}+2 \omega_{\phi} \mathbf{w}_{x} .
\end{aligned}
$$

Finally we have (in matrix form)

$$
\left[\begin{array}{cc}
2 \omega^{x} & -2 \omega^{\phi} \\
2 \omega_{\phi} & 2 \omega_{x}
\end{array}\right]\left[\begin{array}{l}
u_{x} \\
u_{\phi}
\end{array}\right]=\left[\begin{array}{c}
\nabla^{x} \mathbf{w}_{x}-\nabla^{\phi} \mathbf{w}_{\phi}+2 \omega^{x} \mathbf{w}_{x}-2 \omega^{\phi} \mathbf{w}_{\phi} \\
\nabla_{x} \mathbf{w}_{\phi}+\nabla_{\phi} \mathbf{w}_{x}+2 \omega_{x} \mathbf{w}_{\phi}+2 \omega_{\phi} \mathbf{w}_{x}
\end{array}\right]
$$

Let us denote $A:=\left[\begin{array}{cc}2 \omega^{x} & -2 \omega^{\phi} \\ 2 \omega_{\phi} & 2 \omega_{x}\end{array}\right]$. Hence

$$
A^{-1}=\frac{1}{4\left(\omega^{x} \omega_{x}+\omega^{\phi} \omega_{\phi}\right)}\left[\begin{array}{cc}
2 \omega_{x} & 2 \omega^{\phi} \\
-2 \omega_{\phi} & 2 \omega^{x}
\end{array}\right]=\frac{1}{2\|\omega\|^{2}}\left[\begin{array}{cc}
\omega_{x} & \omega^{\phi} \\
-\omega_{\phi} & \omega^{x}
\end{array}\right] .
$$

Multiplying by $A^{-1}$ we get

$$
\begin{gathered}
u_{x}=\frac{1}{2\|\omega\|^{2}}\left(\omega_{x} \nabla^{x} \mathbf{w}_{x}-\omega_{x} \nabla^{\phi} \mathbf{w}_{\phi}+2 \omega_{x} \omega^{x} \mathbf{w}_{x}-2 \omega_{x} \omega^{\phi} \mathbf{w}_{\phi}+\right. \\
\left.+\omega^{\phi} \nabla_{x} \mathbf{w}_{\phi}+\omega^{\phi} \nabla_{\phi} \mathbf{w}_{x}+2 \omega_{x} \omega^{\phi} \mathbf{w}_{\phi}+2 \omega_{\phi} \omega^{\phi} \mathbf{w}_{x}\right)
\end{gathered}
$$


or in simpler form

$$
u_{x}=\frac{1}{2\|\omega\|^{2}}\left(\omega^{x} \nabla_{x} \mathbf{w}_{x}-\omega_{x} \nabla^{\phi} \mathbf{w}_{\phi}+\omega^{\phi} \nabla_{x} \mathbf{w}_{\phi}+\omega^{\phi} \nabla_{\phi} \mathbf{w}_{x}+2\|\omega\|^{2} \mathbf{w}_{x}\right)
$$

Similarly, component $\phi$ :

$$
\begin{gathered}
u_{\phi}=\frac{1}{2\|\omega\|^{2}}\left(\omega_{\phi} \nabla^{\phi} \mathbf{w}_{\phi}-\omega_{\phi} \nabla^{x} \mathbf{w}_{x}+2 \omega_{\phi} \omega^{\phi} \mathbf{w}_{\phi}-2 \omega_{\phi} \omega^{x} \mathbf{w}_{x}+\right. \\
\left.+\omega^{x} \nabla_{x} \mathbf{w}_{\phi}+\omega^{x} \nabla_{\phi} \mathbf{w}_{x}+2 \omega^{x} \omega_{x} \mathbf{w}_{\phi}+2 \omega^{x} \omega_{\phi} \mathbf{w}_{x}\right)
\end{gathered}
$$

or

$$
u_{\phi}=\frac{1}{2\|\omega\|^{2}}\left(\omega_{\phi} \nabla^{\phi} \mathbf{w}_{\phi}-\omega_{\phi} \nabla^{x} \mathbf{w}_{x}+\omega^{x} \nabla_{x} \mathbf{w}_{\phi}+\omega^{x} \nabla_{\phi} \mathbf{w}_{x}+2\|\omega\|^{2} \mathbf{w}_{\phi}\right) \text {. }
$$

Equations (48) and (49) we can rewrite in covariant form:

$$
u_{A}=\frac{1}{2\|\omega\|^{2}}\left(\omega^{B} \nabla_{B} \mathbf{w}_{A}+\omega^{B} \nabla_{A} \mathbf{w}_{B}-\omega_{A} \nabla^{B} \mathbf{w}_{B}+2\|\omega\|^{2} \mathbf{w}_{A}\right)
$$

Now, introducing $\Phi_{A}:=\frac{1}{\|\omega\|^{2}} \omega_{A}$ we have

$$
u_{A}=\mathrm{w}_{A}+\frac{1}{2}\left(\Phi^{B} \nabla_{B} \mathrm{w}_{A}+\Phi^{B} \nabla_{A} \mathbf{w}_{B}-\Phi_{A} \nabla^{B} \mathbf{w}_{B}\right) .
$$

Let us notice the following

$$
\Phi^{B} \nabla_{B} \mathbf{w}_{A}=\nabla_{B}\left(\Phi^{B} \mathbf{w}_{A}\right)-\mathrm{w}_{A} \nabla_{B} \Phi^{B}=\nabla_{B}\left(\Phi^{B} \mathbf{w}_{A}\right)-\mathrm{w}_{A}
$$

(from [6] we know that $\nabla_{B} \Phi^{B}=1$ ),

$$
\begin{aligned}
& \Phi^{B} \nabla_{A} \mathbf{w}_{B}=\nabla_{A}\left(\Phi^{B} \mathbf{w}_{B}\right)-\mathrm{w}_{B} \nabla_{A} \Phi^{B}=\nabla_{A}\left(\Phi^{B} \mathbf{w}_{B}\right)-\mathbf{w}^{B} \nabla_{A} \Phi_{B}, \\
& \Phi_{A} \nabla^{B} \mathbf{w}_{B}=\nabla^{B}\left(\Phi_{A} \mathbf{w}_{B}\right)-\mathbf{w}_{B} \nabla^{B} \Phi_{A}=\nabla_{B}\left(\Phi_{A} \mathbf{w}^{B}\right)-\mathrm{w}^{B} \nabla_{B} \Phi_{A} .
\end{aligned}
$$

The above Eqs. (50), (51), (52) and (53) imply

$u_{A}=\mathbf{w}_{A}+\frac{1}{2}\left[\nabla_{B}\left(\Phi^{B} \mathbf{w}_{A}-\Phi_{A} \mathbf{w}^{B}\right)+\nabla_{A}\left(\Phi^{B} \mathbf{w}_{B}\right)+\mathbf{w}^{B}\left(\nabla_{B} \Phi_{A}-\nabla_{A} \Phi_{B}\right)-\mathbf{w}_{A}\right]$.

From [6] we know that $\varepsilon^{A B} \nabla_{B} \Phi_{A}=0$, hence $\nabla_{B} \Phi_{A}-\nabla_{A} \Phi_{B}=0$, and we obtain formula (36): $u_{A}=\frac{1}{2}\left[\mathrm{w}_{A}+\nabla_{A}\left(\Phi^{B} \mathrm{w}_{B}\right)+\nabla_{B}\left(\Phi^{B} \mathrm{w}_{A}-\Phi_{A} \mathrm{w}^{B}\right)\right]$. 


\section{B.2 Equations for $\mathrm{w}_{A}$}

The trace and curl of $u_{A}$ gives:

$$
\begin{aligned}
& \nabla^{A} \mathbf{w}_{A}+2 \omega^{A} \mathbf{w}_{A}+\nabla^{A} u_{A}=0, \\
& \varepsilon^{A B} \nabla_{B} u_{A}=0 .
\end{aligned}
$$

Using formula

$$
u_{A}=\frac{1}{2}\left[\mathrm{w}_{A}+\nabla_{A}\left(\Phi^{B} \mathrm{w}_{B}\right)+\nabla_{B}\left(\Phi^{B} \mathrm{w}_{A}-\Phi_{A} \mathbf{w}^{B}\right)\right]
$$

and Eq. (54) we obtain

$$
\begin{aligned}
& \nabla^{A} \mathbf{w}_{A}+2 \omega^{A} \mathbf{w}_{A}+\frac{1}{2} \nabla^{A} \mathbf{w}_{A}+\frac{1}{2} \nabla^{A} \nabla_{A}\left(\Phi^{B} \mathbf{w}_{B}\right)+\frac{1}{2} \nabla^{A} \nabla_{B}\left(\Phi^{B} \mathbf{w}_{A}-\Phi_{A} \mathbf{w}^{B}\right)=0 \\
& \Delta\left(\Phi^{B} \mathbf{w}_{B}\right)+3 \nabla^{A} \mathbf{w}_{A}+4 \omega^{A} \mathbf{w}_{A}+\nabla^{A} \nabla^{B}\left(\Phi_{B} \mathbf{w}_{A}-\Phi_{A} \mathbf{w}_{B}\right)=0
\end{aligned}
$$

Moreover, $\nabla^{A} \nabla^{B}\left(\Phi_{B} \mathbf{W}_{A}-\Phi_{A} \mathbf{w}_{B}\right)=0$, because

$$
\nabla_{C} \nabla_{D} t^{A B}-\nabla_{D} \nabla_{C} t^{A B}=R_{E C D}^{A} t^{E B}+R_{E C D}^{B} t^{A E},
$$

where by $R^{A}{ }_{B C D}$ we denote Riemann curvature tensor. We have

$$
\nabla_{A} \nabla_{B} t^{A B}-\nabla_{B} \nabla_{A} t^{A B}=R_{E B} t^{E B}-R_{E A} t^{A E},
$$

where by $R_{A B}$ we denote Ricci tensor. The symmetry of Ricci

$$
\nabla_{A} \nabla_{B} t^{A B}-\nabla_{B} \nabla_{A} t^{A B}=R_{E B} t^{E B}-R_{A E} t^{A E}=0
$$

implies

$$
\begin{aligned}
& \nabla^{A} \nabla^{B}\left(\Phi_{B} \mathbf{w}_{A}-\Phi_{A} \mathbf{w}_{B}\right)=\nabla^{B} \nabla^{A}\left(\Phi_{B} \mathbf{W}_{A}-\Phi_{A} \mathbf{W}_{B}\right)= \\
& =\nabla^{A} \nabla^{B}\left(\Phi_{A} \mathbf{W}_{B}-\Phi_{B} \mathbf{W}_{A}\right)=-\nabla^{A} \nabla^{B}\left(\Phi_{B} \mathbf{W}_{A}-\Phi_{A} \mathbf{W}_{B}\right) .
\end{aligned}
$$

Hence $\nabla^{A} \nabla^{B}\left(\Phi_{B} \mathbf{W}_{A}-\Phi_{A} \mathbf{W}_{B}\right)=0$ and we obtain (35)

$$
\Delta\left(\Phi^{B} \mathbf{w}_{B}\right)+3 \nabla^{A} \mathbf{w}_{A}+4 \omega^{A} \mathbf{w}_{A}=0 .
$$

Using formula (56) and Eq. (55) we get

$$
\varepsilon^{A B} \nabla_{B} \mathbf{w}_{A}+\varepsilon^{A B} \nabla_{B} \nabla^{C}\left(\Phi_{C} \mathbf{w}_{A}-\Phi_{A} \mathbf{w}_{C}\right)+\varepsilon^{A B} \nabla_{B} \nabla_{A}\left(\Phi^{B} \mathbf{w}_{B}\right)=0 .
$$

Vanishing torsion gives $\varepsilon^{A B} \nabla_{B} \nabla_{A}\left(\Phi^{B} \mathrm{w}_{B}\right)=0$, hence

$$
\varepsilon^{A B} \nabla_{B} \mathbf{w}_{A}+\varepsilon^{A B} \nabla_{B} \nabla^{C}\left(\Phi_{C} \mathbf{w}_{A}-\Phi_{A} \mathbf{w}_{C}\right)=0 .
$$


Moreover, $\Phi_{C} \mathbf{w}_{A}-\Phi_{A} \mathbf{w}_{C}=\varepsilon_{A C} \varepsilon^{D E} \Phi_{D} \mathbf{w}_{E}$ implies

$$
\varepsilon^{A B} \nabla_{B} \mathrm{w}_{A}+\varepsilon^{A B} \varepsilon_{A C} \nabla_{B} \nabla^{C}\left({ }_{\varepsilon}^{D E} \Phi_{D} \mathrm{w}_{E}\right) .
$$

Using identity $\varepsilon^{A B} \varepsilon_{A C}=-\delta^{B} C$, we get

$$
\varepsilon^{A B} \nabla_{B} \mathbf{w}_{A}+\nabla_{C} \nabla^{C}\left(\varepsilon^{A B} \Phi_{B} \mathbf{w}_{A}\right)=0,
$$

and finally we obtain (34)

$$
\Delta\left(\varepsilon^{A B} \Phi_{B} \mathrm{~W}_{A}\right)+\varepsilon^{A B} \nabla_{B} \mathrm{~W}_{A}=0 .
$$

\section{Appendix C: Proof for large $k$}

Stability for the extremal Kerr leads to the following equation:

$$
\partial_{x} a^{2} \partial_{x} v_{k}-\frac{k^{2}}{a^{2}} v_{k}+D v_{k}+\partial_{x}\left(a^{2} B v_{k}\right)+i k C v_{k}=0,
$$

where

- $a^{2}=2 \frac{1-x^{2}}{1+x^{2}}$.

- $v_{k}:[-1,1] \rightarrow \mathbb{C}^{2}$ is the unknown function we are looking for,

- $B=\frac{1}{1+x^{2}}\left[\begin{array}{ll}x & -1 \\ 3 & 3 x\end{array}\right]$,

- $C=\frac{1}{1+x^{2}}\left[\begin{array}{cc}1 & x \\ -3 x & 3\end{array}\right]$,

- $D=\frac{4 a^{2}}{1+x^{2}}\left[\begin{array}{ll}0 & 0 \\ 0 & 1\end{array}\right]$.

Theorem 5 Equation (57) has no solutions for $|k|>8$.

Proof For functions $f, g:[-1,1] \rightarrow \mathbb{C}^{2}$ let us define a standard scalar product:

$$
(f \mid g)=\int_{-1}^{1} \bar{f}^{T} g \mathrm{~d} x
$$

Let us consider an operator $X:=a \frac{d}{d x}$ and its hermitian conjugate $X^{*}=-\frac{d}{d x} a$. The Eq. (57) takes the form:

$$
X^{*} X v_{k}+\frac{k^{2}}{a^{2}} v_{k}+X^{*}\left(a B v_{k}\right)-i k C v_{k}-D v_{k}=0
$$


The left-hand side we denote by $L v_{k}$, where $L$ is a linear operator and $v_{k} \in \operatorname{ker} L$, i.e. $L v_{k}=0$. For $\left(v_{k} \mid L v_{k}\right)$ we have:

$0=\left(v_{k} \mid L v_{k}\right)=\left\|X v_{k}\right\|^{2}+k^{2}\left\|\frac{1}{a} v_{k}\right\|+\left(X v_{k} \mid a B v_{k}\right)-i k\left(\frac{1}{a} v_{k} \mid a C v\right)-\left(v_{k} \mid D v_{k}\right)$

Introducing real numbers $x:=\frac{\left\|X v_{k}\right\|}{\left\|v_{k}\right\|}, y:=\frac{\left\|\frac{1}{a} v_{k}\right\|}{\left\|v_{k}\right\|}$ we obtain:

$$
x^{2}\left\|v_{k}\right\|^{2}+k^{2} y^{2}\left\|v_{k}\right\|^{2}=-\left(X v_{k} \mid a B v_{k}\right)+i k\left(\frac{1}{a} v_{k} \mid a C v_{k}\right)+\left(v_{k} \mid D v_{k}\right),
$$

and absolute value one can estimate as follows:

$$
x^{2}\left\|v_{k}\right\|^{2}+k^{2} y^{2}\left\|v_{k}\right\|^{2} \leq\left|\left(X v_{k} \mid a B v_{k}\right)\right|+|k|\left|\left(\frac{1}{a} v_{k} \mid a C v_{k}\right)\right|+\left|\left(v_{k} \mid D v_{k}\right)\right| .
$$

From Cauchy-Schwarz inequality

$$
x^{2}\left\|v_{k}\right\|^{2}+k^{2} y^{2}\left\|v_{k}\right\|^{2} \leq x\left\|v_{k}\right\|\left\|a B v_{k}\right\|+|k| y\left\|v_{k}\right\|\left\|a C v_{k}\right\|+\left\|v_{k}\right\|\left\|D v_{k}\right\|,
$$

and from $\|A v\| \leq\|A\|\|v\|$ we get:

$$
x^{2}\left\|v_{k}\right\|^{2}+k^{2} y^{2}\left\|v_{k}\right\|^{2} \leq(x\|a B\|+|k| y\|a C\|+\|D\|)\left\|v_{k}\right\|^{2} .
$$

Hence

$$
x^{2}+k^{2} y^{2} \leq x\|a B\|+|k| y\|a C\|+\|D\|
$$

or in an equivalent form:

$$
\left(x-\frac{\|a B\|}{2}\right)^{2}+\left(|k| y-\frac{\|a C\|}{2}\right)^{2} \leq\|D\|+\frac{\|a B\|^{2}+\|a C\|^{2}}{4} .
$$

Positivity of $\left(x-\frac{\|a B\|}{2}\right)^{2}$ gives

$$
\left(|k| y-\frac{\|a C\|}{2}\right)^{2} \leq\|D\|+\frac{\|a B\|^{2}+\|a C\|^{2}}{4}
$$

and

$$
|k| \leq \frac{\|a C\|+\sqrt{4\|D\|+\|a B\|^{2}+\|a C\|^{2}}}{2 y} .
$$


Definition of $y=\frac{\left\|\frac{1}{a} v_{k}\right\|}{\left\|v_{k}\right\|}$ and $a \leq \sqrt{2}$ gives $y \geq \frac{1}{\sqrt{2}}$, hence

$$
|k| \leq \frac{\|a C\|+\sqrt{4\|D\|+\|a B\|^{2}+\|a C\|^{2}}}{\sqrt{2}} .
$$

A simple computation gives $\|D\|=8,\|a B\|=\sqrt{6},\|a C\|=3 \sqrt{2}$. Finally

$$
|k| \leq \frac{3 \sqrt{2}+\sqrt{32+6+18}}{\sqrt{2}}=3+\sqrt{28} \approx 8.29
$$

but $k$ is integer hence $|k| \leq 8$.

\section{Appendix D: Conformal vector field for extremal Kerr}

We are looking for a vector field $X$ in the following form:

$$
X=A(x) \cos \phi \partial_{x}+B(x) \sin \phi \partial_{\phi} .
$$

In coordinates $(x, \phi)$ the metric tensor $\left(g_{A B}\right)=\left(\begin{array}{cc}m^{2} \frac{1+x^{2}}{1-x^{2}} & 0 \\ 0 & 4 m^{2} \frac{1-x^{2}}{1+x^{2}}\end{array}\right)$, hence

$$
X_{x}=A m^{2} \frac{1+x^{2}}{1-x^{2}} \cos \phi, \quad X_{\phi}=4 B m^{2} \frac{1-x^{2}}{1+x^{2}} \sin \phi
$$

The CVF equation

$$
\nabla_{A} X_{B}+\nabla_{B} X_{A}=\nabla_{C} X^{C} g_{A B}
$$

applied to our field $X$ reduces to

$$
\begin{aligned}
& \nabla_{x} X_{x}=\left(\frac{1+x^{2}}{1-x^{2}} A^{\prime}+\frac{2 x}{\left(1-x^{2}\right)^{2}} A\right) m^{2} \cos \phi \\
& \nabla_{\phi} X_{\phi}=4 m^{2}\left(\frac{1-x}{1+x^{2}} B-\frac{2 x}{\left(1+x^{2}\right)^{2}} A\right) \cos \phi \\
& \nabla_{\phi} X_{x}+\nabla_{x} X_{\phi}=\left(4 \frac{1-x^{2}}{1+x^{2}} B^{\prime}-\frac{1+x^{2}}{1-x^{2}} A\right) m^{2} \sin \phi, \\
& \nabla_{C} X^{C}=\left(A^{\prime}+B\right) \cos \phi
\end{aligned}
$$

where $A^{\prime}:=\frac{d A}{d x}$. 
They can be written in an equivalent form:

$$
\begin{aligned}
& \left(\frac{1+x^{2}}{1-x^{2}} A^{\prime}+\frac{2 x}{\left(1-x^{2}\right)^{2}} A\right) m^{2} \cos \phi=\frac{1}{2} m^{2} \frac{1+x^{2}}{1-x^{2}}\left(A^{\prime}+B\right) \cos \phi, \\
& 4 m^{2}\left(\frac{1-x}{1+x^{2}} B-\frac{2 x}{\left(1+x^{2}\right)^{2}} A\right) \cos \phi=2 m^{2} \frac{1-x^{2}}{1+x^{2}}\left(A^{\prime}+B\right) \cos \phi, \\
& 4 \frac{1-x^{2}}{1+x^{2}} B^{\prime}-\frac{1+x^{2}}{1-x^{2}} A=0,
\end{aligned}
$$

and finally we obtain system of ODE's:

$$
\begin{aligned}
& B=A^{\prime}+\frac{4 x}{1-x^{4}} A, \\
& 4 B^{\prime} \frac{1-x^{2}}{1+x^{2}}=A \frac{1+x^{2}}{1-x^{2}},
\end{aligned}
$$

which leads to the second order ODE for the function $B$ :

$$
\left[\partial_{x}^{2}-\frac{4 x}{1-x^{4}} \partial_{x}-\frac{1}{4}\left(\frac{1+x^{2}}{1-x^{2}}\right)^{2}\right] B(x)=0
$$

and $A(x)=4\left(\frac{1-x^{2}}{1+x^{2}}\right)^{2} B^{\prime}(x)$. We get the following solution:

$$
B(x)=C_{1} \cosh \left[\frac{1}{2}\left(x+\log \frac{1-x}{1+x}\right)\right]+C_{2} \sinh \left[\frac{1}{2}\left(x+\log \frac{1-x}{1+x}\right)\right] .
$$

If we assume that the field $X$ vanishes at one "pole" $(x= \pm 1)$ we obtain the relation for constants $C_{i}: C_{2}= \pm C_{1}$. For $C_{2}=-C_{1}$ we have:

$$
B(x)=C \sqrt{\frac{1+x}{1-x}} e^{-\frac{1}{2} x}, \quad A(x)=2 C \sqrt{\frac{1+x}{1-x}} \frac{1-x^{2}}{1+x^{2}} e^{-\frac{1}{2} x} .
$$

Finally the CVF $X$ takes the form:

$$
X=C \sqrt{\frac{1+x}{1-x}} e^{-\frac{1}{2} x}\left(2 \frac{1-x^{2}}{1+x^{2}} \cos \phi \partial_{x}+\sin \phi \partial_{\phi}\right)
$$

\section{References}

1. Ashtekar, A., Beetle, C., Lewandowski, J.: Geometry of generic isolated horizons. Class. Quantum Gravity 19, 1195-1225 (2002)

2. Amsel, A.J., Horowitz, G.T., Marolf, D., Roberts, M.M.: Uniqueness of extremal Kerr and KerrNewman black holes. Phys. Rev. D 81, 024033, (2010) arXiv:0906.2367v2 [gr-qc]

3. Bardeen, J., Horowitz, G. T.: The extreme kerr throat geometry: a vacuum analog of $A d S_{2} \times S^{2}$. Phys. Rev. D 60, 104030 (1999) arXiv:hep-th/9905099v1 
4. Chruściel, P., Reall, H.S., Tod, P.: On non-existence of static vacuum black holes with degenerate components of the event horizon, NI05066-GMR. Class. Quantum Gravity 23, 549-554 (2006)

5. Hollands, S., Ishibashi, A.: All vacuum near horizon geometries in arbitrary dimensions, arXiv:0909.3462v2 [gr-qc]

6. Jezierski, J.: On the existence of Kundt's metrics and degenerate (or extremal) killing horizons. Class. Quantum Gravity 26, 035011 (11pp) (2009)

7. Kundt, W.: The plane-fronted gravitational Waves. Z. Phys. 163, 77-86 (1961)

8. Kundt, W., Trümper, M.: Beitraege zur Theorie der Gravitations-Strahlungsfelder. Akad. Wiss. Lit. Mainz Ahandl. Math.-Nat. Kl. 12, 967-1000 (1962)

9. Kunduria, H.K., Lucietti, J.: A classification of near-horizon geometries of extremal vacuum black holes, arXiv:0806.2051v2 [hep-th] 22 Jul 2008

10. Lewandowski, J., Pawłowski, T.: Extremal isolated horizons: a local uniqueness theorem. Class. Quantum Gravity 20, 587-606 (2003)

11. Pawłowski, T., Lewandowski, J., Jezierski, J.: Spacetimes foliated by killing horizons, gr-qc/0306107. Class. Quantum Gravity 21, 1237-1251 (2004)

12. Szmytkowski, R.: Closed form of the generalized green's function for the Helmholtz operator on the two-dimensional unit sphere. J. Math. Phys. 47, 063506 (2006) 